\title{
Antibiotic Resistant, Virulence-associated Genes, Biofilm and Efflux Pump Gene Expression and Molecular Typing of Klebsiella Pneumoniae Strains Recovered from Clinical Samples
}

\author{
Amir Mirzaie \\ Baghiatollah University of Medical Sciences: Baqiyatallah University of Medical Sciences \\ Reza Ranjbar ( $\nabla$ ranjbarre@gmail.com ) \\ Baghiatollah University of Medical Sciences: Baqiyatallah University of Medical Sciences
}

Research

Keywords: Klebsiella pneumoniae, antibiotic resistant, efflux pumps, Biofilm, gene expression, rep-PCR

Posted Date: October 28th, 2020

DOI: https://doi.org/10.21203/rs.3.rs-96879/v1

License: (c) (i) This work is licensed under a Creative Commons Attribution 4.0 International License. Read Full License 


\section{Abstract}

\section{Background}

Multidrug-resistant (MDR) Klebsiella pneumoniae strains are one of the most important life-threatening nosocomial pathogens. In the current study, antibiotic resistant, virulence-associated genes, gene expression of efflux pumps and biofilm genes as well as molecular typing of $K$. pneumoniae strains were investigated. A total of 505 clinical specimens were collected from hospitalized patients and $K$. pneumoniae strains were isolated by standard microbiological methods. Antibiotic resistant profile, prevalence of virulence associated genes, biofilm and efflux pump genes were investigated. The gene expression analysis of biofilm and efflux pump genes were analused quantitative Real Time PCR. Moreover, molecular typing of $K$. pneumoniae strains using Repetitive element sequence-based PCR (rep-PCR) technique was also carried out.

Results

One hundred K. pneumoniae strains out of 500 clinical samples were isolated and the highest prevalence of resistance was observed against ciprofloxacin (75\%), Trimethoprim-sulfamethoxazole (73\%) and Nitrofurantoin (38\%). Virulence associated genes including entB, traT and $r m p A$ were found in $80 \%, 62 \%$ and $48 \%$, respectively. Gene prevalence for biofilm association gene including $m r k A$, fimH and $m r k D$ were $42 \%$ for all genes. The $A c r A B, T o / C$ and $m d t K$ efflux pump genes were observed in $41 \%, 33 \%$ and $26 \%$, respectively. In addition, most MDR strains formed biofilm, as well as, $A c r A B$ efflux pump and $m r k A$ biofilm gene expression was up-regulated in MDR K. pneumoniae strains and a significant statistically association was also observed between MDR strains and high expression of efflux pump and biofilm genes. In addition, the K. pneumoniae strains differentiated into 11 different genetic clusters by rep-PCR analysis.

Conclusions

High prevalence of resistance, presence of diver's virulence factors and high level of efflux pump and biofilm gene expression in diverse clones of $K$. pneumoniae strains pose an important public health issue.

\section{Background}

Klebsiella pneumoniae is a Gram-negative pathogen belonging to the Enterobacteriaceae family [1]. $K$. pneumoniae can cause various infections such as urinary tract infections, pneumonia and bloodstream infections and it is considered as an important nosocomial pathogen in Iran [2]. K. pneumoniae has recently attracted the attention of researchers due to the increased severity of infections, antibiotic resistance and treatment deficiencies $[3,4]$. The increase in multidrug-resistant (MDR) K. pneumoniae in recent years is mainly due to the indiscriminate use of antibiotics to treat and prevent infections caused by this bacterium [5]. $K$. pneumoniae uses a variety of mechanisms to resist antibiotics [6]. One of the important mechanisms for creating the MDR is efflux pump systems and biofilm formation capacity [7]. Efflux pumps are protein based structure which capable extrude the different toxic substances out of cells and the AcrAB-TolC efflux pump system which belongs to the Resistance Nodulation Division (RND) efflux pump which is an important cause of MDR of K. pneumoniae strains occurrence [8-11]. The AcrAB-TolC efflux pump is composed a periplasmic component (AcrA), a transporter located in the inner membrane (AcrB) and an outer membrane compartment (TolC). The AcrAB-TolC efflux pump plays an important role in developing resistance to various antibiotics such 
quinolones, tetracycline, and chloramphenicol in MDR strains of $K$. pneumoniae [12]. The ability to form biofilms in K. pneumoniae strains, allows it to protect against the host immune system and antibiotics in MDR isolates and there are a lot of biofilm related genes including mrk (type 3 fimbriae), fimH-1 (type 1 fimbrial adhesion) $[13,14]$. Studies show that the efflux pump plays an important role in antibiotic resistance and biofilm formation [15]. There are several studies showed a correlation of $K$. pneumoniae antibiotic resistance, efflux pump and biofilm formation ability [16]. In the study of Subramanian et al, 2012, indicated that $80 \%$ of biofilm forming isolates from 100 clinical samples showed an MDR phenotype [17]. Various Virulence factors such as those encoding regulators of mucoid phenotype $A(r m p A)$, bacteriocin biosynthesis [enterobactin (entB), and serum resistance-associated outer membrane lipoprotein (traT) play an important role in the pathogenicity of $K$. pneumoniae strains [18].

Molecular typing of $K$. pneumoniae strains can be used to prevent infection in the hospital and also to find the dominant genotype among the isolates can be useful in finding the source of infection and applying prevention

protocols $[19,20]$. Due to the high prevalence of MDR strains of $K$. Pneumoniae in Iran, the aim of this study was to investigate the antibiotic resistance profile and distribution of virulence genes in $K$. Pneumoniae strains, efflux pump and biofilm gene expression, as well as, molecular typing of $K$. pneumoniae strains using Repetitive element sequence-based PCR (rep-PCR).

\section{Methods}

\section{Bacterial isolates and identification}

A total of 505 clinical specimens including urine, blood, sputum and cerebrospinal fluid (CSF) were collected from hospitalized patients were admitted to Baghiatallah and Imam Khomeini hospitals in Iran from January 2018 to July 2019. The K. pneumoniae strains were identified using conventional microbiological tests including catalase, MR-VP (methyl red - Voges Proskauer), lysine iron agar, Kligler agar, phenylalanine agar, urea agar, SIM (sulfide, indole, motility), blood agar, and MacConkey agar [21]. The isolated strains were stored at $-20^{\circ} \mathrm{C}$ in brain heart infusion broth containing $20 \%$ glycerol for further investigation.

\section{Antimicrobial susceptibility test}

Antibiotic susceptibility of $K$. pneumoniae strains was evaluated according to the Clinical and Laboratory Standards Institute (CLSI) standard based on to Kirby-Bauer disk diffusion method [22]. The antimicrobial susceptibility assays to 17 antibiotics were performed using commercially available antibiotics including ceftazidime $(30 \mu \mathrm{g})$, cefotaxime $(30 \mu \mathrm{g})$, cefoxitin $(30 \mu \mathrm{g})$, ceftriaxone $(30 \mu \mathrm{g})$, tobramycin $(10 \mu \mathrm{g})$, gentamicin $(10 \mu \mathrm{g})$, streptomycin $(30 \mu \mathrm{g})$, nalidixic acid $(30 \mu \mathrm{g})$, ciprofloxacin $(5 \mu \mathrm{g})$, Imipenem $(10 \mu \mathrm{g})$, co-trimoxazole $(1.25 / 23.75 \mu \mathrm{g})$, chloramphenicol $(30 \mu \mathrm{g})$, Amoxicillin/Clavulanic acid (AMC, 20/10 $\mu \mathrm{g})$, Meropenem $(10 \mu \mathrm{g})$, clindamycin $(30 \mu \mathrm{g})$, polymixin B $(10 \mu \mathrm{g})$, tetracycline $(30 \mu \mathrm{g})$ (MAST, Group Ltd., Merseyside, UK). The plates were incubated at $37^{\circ} \mathrm{C}$ for 24 hours and subsequently, the inhibition zone diameters were recorded in millimeter and interpretation was done based on CLSI. K. pneumoniae ATCC 13883 was used as a control in this study. Multidrug-resistant (MDR) strains were identified based on their resistance to at least one drug in three different groups of antibiotics. 


\section{Phenotypic detection of efflux pump}

The phenotypic and qualitative detection of the efflux pump in Klebsiella pneumoniae strains was performed by Cartwheel method. Briefly, the plates of Muller Hinton Agar culture media containing ethidium bromide were prepared and the culture medium was divided into 8 parts and the bacteria in $0.5 \mathrm{McF}$ arland turbidity concentration were streaked on plates. After $24 \mathrm{~h}$ of incubation at $37^{\circ} \mathrm{C}$, the plates were studied under UV transilluminator. The strains that had efflux pumps did not show emission of fluorescence [23].

\section{Phenotypic detection of biofilm formation}

Phenotypic detection of biofilm formation was performed using Congo red agar test. Briefly, the K. pneumoniae strains was cultured in Brain Heart Infusion agar enriched with $5 \%(w / v)$ sucrose and Congo red based on Freeman et al. 1989. The K. pneumoniae strains which formed biofilm exhibited a dry dark crystalline colonies and considered as exopolysaccharides producers [24].

\section{Quantitative detection of biofilm}

Quantitative biofilm detection test was performed by plate microtiter method in 96 house plate. In summary, first $100 \mu$ l of 24-hour culture of strains with $O D=0.1$ were added into wells. After $24 \mathrm{~h}$ incubation, each well was washed twice with PBS and then, stained with crystal violet for 15 min.

Finally, the stained cells were solubilized in $33 \%(\mathrm{v} / \mathrm{v})$ acetic acid and their adsorption was read at $570 \mathrm{~nm}$ OD $570 \mathrm{~nm}$. Based on the type of biofilm, the strains of biofilm former were divided into three categories: strong biofilm, moderate biofilm, weak biofilm and negative biofilm producers. In addition, the standard strain $K$. pneumoniae ATCC 13833 and LB broth was considered as a positive and negative control, respectively [25].

\section{Molecular detection of virulence associated genes}

The genomic DNA of the strains was extracted by the DNA extraction kit (Bioneer, Korea) according to the manufacturer's protocol. The frequency of entB, Trat and $r m p A$ virulence gene, $m d t k$, tolC and $A c r A B$ efflux pump, fimH-1, $m r k A$ and $m r k B$ biofilm associated genes were detected by PCR. The PCR conditions were as follow: Initial denaturing at $94{ }^{\circ} \mathrm{C}$ for 5 min followed by 30 cycles, each cycle contained 1 min at $94{ }^{\circ} \mathrm{C}$ for denaturation, 30 second for annealing (Table 1 ) and $60 \mathrm{~S}$ for extension steps and finally one cycle for final extension at $72{ }^{\circ} \mathrm{C}$ for $10 \mathrm{~min}$. The primer of target genes are given in Table 1. 
Table 1

The primer sequences of target genes which used in this study.

\begin{tabular}{|c|c|c|c|}
\hline Target gene & Primer sequence $\left(5^{\prime}\right.$ to $\left.3^{\prime}\right)$ & Annealing Temperature $\left({ }^{\circ} \mathrm{C}\right)$ & Reference \\
\hline \multirow[t]{2}{*}{$A c r A B$} & F ATCAGCGGCCGGATTGGTAAA & 53 & {$[26]$} \\
\hline & R CGGGTTCGGGAAAATAGCGCG & & \\
\hline \multirow[t]{2}{*}{ TolC } & F ATCAGCAACCCCGATCTGCGT & 51 & {$[26]$} \\
\hline & R CCGGTGACTTGACGCAGTCCT & & \\
\hline \multirow[t]{2}{*}{ Mdtk } & F GCGCTTAACTTCAGCTCA & 43 & {$[26]$} \\
\hline & R GATGATAAATCCACACCAGAA & & \\
\hline \multirow[t]{2}{*}{$m r k A$} & F ACGTCTCTAACTGCCAGGC & 55 & {$[16]$} \\
\hline & R TAGCCCTGTTGTTTGCTGGT & & \\
\hline \multirow[t]{2}{*}{$m r k D$} & F CCACCAАСТАTTСССТCGAA & 43 & {$[27,28]$} \\
\hline & R ATGGAACCCACATCGACATT & & \\
\hline \multirow[t]{2}{*}{ fimH } & F GCCAACGTCTACGTTAACCTG & 43 & {$[26]$} \\
\hline & R ATATTTCACGGTGCCTGAAAA & & \\
\hline \multirow[t]{2}{*}{ entB } & F CTGCTGGGAAAAGCGATTGTC & 49 & {$[26]$} \\
\hline & R AAGGCGACTCAGGAGTGGCTT & & \\
\hline \multirow[t]{2}{*}{$\operatorname{traT}$} & F GGTGTGGTGCGATGAGCACAG & 55 & {$[28]$} \\
\hline & R CACGGTTCAGCCATCCCTGAG & & \\
\hline \multirow[t]{2}{*}{ rmpA } & F ACTGGGCTACCTCTGCTTCA & 53 & {$[29]$} \\
\hline & R CTTGCATGAGCCATCTTTCA & & \\
\hline \multirow[t]{2}{*}{$16 S$ rRNA } & F AGCCGACCTGAGAGGGTGA & 55 & {$[30]$} \\
\hline & R TCTGGACCGTGTCTCAGTTCC & & \\
\hline
\end{tabular}

\section{Gene expression analysis of $A c r A B$ and $m r k A$ gene}

AcrAB efflux pump and $m r k A$ biofilm gene expression analysis was done using quantitative Real Time PCR method in K. pneumoniae strains. Briefly, $100 \mu$ l of each bacterium was added into 96 well plate and incubated for $18 \mathrm{~h}$ at $37^{\circ} \mathrm{C}$ aerobically. Then, each well was washed using PBS and adherent cells were scraped off using LB broth. Subsequently, total RNA of collected strains were extracted using an RNA extraction kit (Qiagen, USA) according to instruction protocol. 
The extracted RNA was converted to complementary DNA (cDNA) by a cDNA synthesis kit (Fermentase, Lithuania) based on manufacturer's instructions. In order to perform the Real Time PCR, each CDNA was used as a template in $20 \mu \mathrm{l}$ final volume containing $2 \mu \mathrm{l} \mathrm{cDNA}, 10$ pmol of each primer (Table 1), and $10 \mu \mathrm{l}$ Power SYBR Green PCR Master Mix (Applied Biosystems) using Bioneer Real-Time PCR equipment (Korea). The $16 \mathrm{~S}$ rRNA was used as a housekeeping gene to normalize the levels of mRNA expression and the relative expression of $A c r A B$ efflux pump gene was calculated using $\triangle \triangle C$ T method.

\section{Molecular typing of MDR K. pneumoniae strains}

Molecular typing of $K$. pneumoniae strains was done using the Repetitive element sequence-based PCR (repPCR) method. The rep-PCR was performed using two following primers: Forward: REP1 5'- III ICG ICG ICA TCI GGC-3' Reverse: REP2 5'- ICG ICT TATCIG GCC TAC-3' as described previously. The PCR amplification products were visualized using electrophoresis on $1.5 \%$ agarose gel and stained with safe red. Finally, the banding patterns and size were determined via Image Lab 4.0. The obtaining results were analyzed by gel compare II software using Dice correlation coefficient and the UPGMA method [31].

\section{Statistical Analysis}

All tests of this study are repeated three times and one way ANNOVA test was used for statistical analysis. In addition, $\mathrm{P}<0.05$ was also considered significant.

\section{Results}

\section{Isolation and antimicrobial susceptibility pattern of $K$. pneumoniae strains}

A total of $100 \mathrm{~K}$. pneumoniae isolates were collected from 505 clinical samples. The $100 \mathrm{~K}$. pneumoniae strains were recovered from specimens of urine $(n=70)$, blood $(n=20)$, Sputum $(n=6)$ and cerebrospinal fluid $(C S F, n=4)$ based on routine microbiological methods. Among 100 clinical strains of $K$. pneumoniae, 75 (75\%), 73 (73\%) and $68(68 \%)$ of strains were resistant to ciprofloxacin, Trimethoprim-sulfamethoxazole and Nitrofurantoin, respectively and $28(28 \%), 52(52 \%)$ strains were susceptible to streptomycin and imipenem, respectively (Table 2). In addition, 92 (92\%) strains revealed MDR phenotypes and most MDR strains were resistant to imipenem, meropenem and beta-lactam antibiotics. The studied strains were categorized into 25 antimicrobial resistant patterns (Table 2).

\section{Phenotypic detection of biofilm formation and efflux pump}

Phenotypic detection of biofilm formation was done using Congo red agar test and our results showed that $77 \%$ (77 strains) of isolates exhibited black colonies, which presumably indicate biofilm formation (Fig 1A). The other strains formed white colonies which reflecting no biofilm formation. Moreover, the Cartwheel results showed that $90 \%$ (90 isolates) of strains had efflux pumps (Fig 1B). 
Table 2

Antimicrobial sensitivity, biofilm formation and efflux pump patterns of $K$. pneumoniae strains.

\begin{tabular}{|c|c|c|c|c|c|c|}
\hline Isolates NO. & Antibiotic resistant pattern & $\begin{array}{l}\text { Anti- } \\
\text { biotype } \\
\text { (AB) }\end{array}$ & MDR & $\begin{array}{l}\text { Biofilm } \\
\text { former }\end{array}$ & $\begin{array}{l}\text { Mean OD } \\
570\end{array}$ & $\begin{array}{l}\text { Phenotypic } \\
\text { efflux } \\
\text { pump }\end{array}$ \\
\hline $\begin{array}{l}10,17,23,73 \\
92,98\end{array}$ & $\begin{array}{l}\text { CTX, CAZ, CRO, FOX, NA, CP, } \\
\text { FM, TE, S, GM }\end{array}$ & AB1 & + & + & $2.63 \pm 0.14$ & + \\
\hline 15,20 & $\begin{array}{l}\text { CTX, CAZ, CRO, FOX, NA, CP, } \\
\text { FM, TMP }\end{array}$ & AB2 & + & - & $0.025 \pm 0.03$ & + \\
\hline $1,6,13,25$ & $\begin{array}{l}\text { CTX, CAZ, CRO, FOX, TMP, } \\
\text { GM, S }\end{array}$ & $\mathrm{AB} 3$ & + & + & $2.13 \pm 0.19$ & + \\
\hline $\begin{array}{l}4,12,21,74 \\
77,94\end{array}$ & CAZ, FOX, FM, TE, TMP, S & $\mathrm{AB} 4$ & + & + & $3.52 \pm 0.21$ & + \\
\hline $18,35,49$ & $\begin{array}{l}\text { CTX, CAZ, CRO, FOX, FM, } \\
\text { TMP }\end{array}$ & AB 5 & + & + & $0.76 \pm 0.06$ & + \\
\hline $3,16,22$ & CTX, CAZ, CRO, NA, CP & $A B 6$ & + & + & $0.38 \pm 0.08$ & + \\
\hline $8,45,75$ & $\begin{array}{l}\text { CTX, CAZ, CRO, FOX, NA, CP, } \\
\text { FM, TE }\end{array}$ & $A B 7$ & + & + & $2.69 \pm 0.17$ & + \\
\hline $5,41,76$ & $\begin{array}{l}\text { CTX, CAZ, CRO, FOX, NA, CP, } \\
\text { AMC, CF, S, FM, TMP, IMP, } \\
\text { GM, MEN }\end{array}$ & AB 8 & + & + & $3.4 \pm 0.12$ & + \\
\hline $19,31,72$ & $\begin{array}{l}\text { CTX, CAZ, CRO, NA, CP, FM, } \\
\text { TMP, GM, S }\end{array}$ & $\mathrm{AB} 9$ & + & + & $1.8 \pm 0.07$ & - \\
\hline 7 & AMC, S, CF, TMP & $A B 10$ & + & + & $0.17 \pm 0.16$ & + \\
\hline $9,28,87$ & $\begin{array}{l}\text { FM, CF, AMC, K, GM, TMP, } \\
\text { CP, AN, FOX, CTX, CRO, CAZ }\end{array}$ & AB 11 & + & + & $0.81 \pm 0.007$ & + \\
\hline $24,26,40$ & $\begin{array}{l}\text { S, CF, AMC, FM, IPM, TMP, } \\
\text { MEN, NA, CP }\end{array}$ & AB 12 & + & - & $0.16 \pm 0.003$ & + \\
\hline 14,39 & $\begin{array}{l}\text { CL, TE, PB, AN, TMP, GM, K, } \\
\text { S, FM, AMC, CF }\end{array}$ & AB 13 & + & + & $0.15 \pm 0.006$ & + \\
\hline $41,59,61,93$ & $\mathrm{AMC}, \mathrm{CF}, \mathrm{FM}, \mathrm{PB}, \mathrm{CL}, \mathrm{TE}$ & $A B 14$ & + & + & $0.94 \pm 0.007$ & - \\
\hline 36,43 & $\begin{array}{l}\text { CP, TE, NA, FM, MEN, TMP, } \\
\text { CF, AMC }\end{array}$ & $\mathrm{AB} 15$ & + & + & $0.16 \pm 0.006$ & + \\
\hline $53,81,96$ & $\begin{array}{l}\text { CF, AMC, MEN, TMP, IPM, } \\
\text { CP, NA }\end{array}$ & $\mathrm{AB} 16$ & + & + & $0.20 \pm 0.13$ & + \\
\hline $27,30,62,88$ & $\begin{array}{l}\text { FM, AMC, CF, IPM, AN, MEN, } \\
\text { GM, TMP, CP, TE, NA, CL }\end{array}$ & $\mathrm{AB} 17$ & + & + & $0.19 \pm 0.001$ & + \\
\hline $\begin{array}{l}2,11,60,78 \\
100\end{array}$ & CF, AMC, TMP, CP, TE, NA & AB 18 & + & + & $0.13 \pm 0.006$ & + \\
\hline $50,80,85,86$ & $\begin{array}{l}\text { AMC, FM, CF, CP, TE, NA, } \\
\text { MEN, IPM, TMP, GM, AN }\end{array}$ & AB 19 & + & + & $2.13 \pm 0.003$ & + \\
\hline
\end{tabular}




\begin{tabular}{|c|c|c|c|c|c|c|}
\hline $48,52,58,65$ & $\begin{array}{l}\text { AMC, S, CF, FM, IPM, MEN, } \\
\text { CP, NA }\end{array}$ & AB 20 & + & + & $3.82 \pm 0.009$ & + \\
\hline $\begin{array}{l}33,47,71,56 \\
83,69\end{array}$ & $\begin{array}{l}\text { CF, K, FM, AMC, GM, MEN, } \\
\text { IPM, AN, TMP, NA, TE, CP }\end{array}$ & AB 21 & + & + & $0.19 \pm 0.007$ & + \\
\hline $34,42,99$ & $\begin{array}{l}\text { AMC, CF, FM, IMP, MEN, } \\
\text { TMP, CP, NA, TE }\end{array}$ & AB 22 & + & + & $1.45 \pm 0.008$ & - \\
\hline $\begin{array}{l}44,50,54,7 \\
66,79,97,63 \\
64,70,82\end{array}$ & $\begin{array}{l}\text { CAZ, CRO, FOX, K, FM, AMC, } \\
\text { CF, AN, TMP, GM, IMP, MEN, } \\
\text { CP, TE, NA }\end{array}$ & AB 23 & + & - & $0.02 \pm 0.002$ & + \\
\hline $\begin{array}{l}29,32,51,55 \\
57,68,84\end{array}$ & $\begin{array}{l}\text { CP, TE, NA, TMP, GM, IMP, } \\
\text { MEN, AMC, FM, CF, CAZ, } \\
\text { CTX, CRO, FOX }\end{array}$ & AB 24 & + & - & $0.029 \pm 0.001$ & + \\
\hline $\begin{array}{l}30,89,90,91 \\
37,38,46,95\end{array}$ & $\mathrm{CF}, \mathrm{AMC}$ & AB 25 & - & + & $0.56 \pm 0.001$ & + \\
\hline $\begin{array}{l}\text { CF: Chloramph } \\
\text { CAZ: Ceftazidir } \\
\text { Trimethoprim-s } \\
\text { Kanamycin, CL }\end{array}$ & $\begin{array}{l}\text { ol, AMC: Amoxicillin-clavula } \\
\text { M: Nitrofurantoin, MEN: Me } \\
\text { methoxazole, NA: Nalidixic } \\
\text { damycin, PB: Polymixin B, }\end{array}$ & $\begin{array}{l}\text { acid, FC } \\
\text { enem, II } \\
\text {, TE: Te } \\
\text { trepton }\end{array}$ & & & $\begin{array}{l}\text { eriaxon, CTX } \\
\text { ntamycin, TM } \\
\text { xacin, S: Stre }\end{array}$ & $\begin{array}{l}\text { efotaxime, } \\
\text { omycin, } \mathrm{K} \text { : }\end{array}$ \\
\hline
\end{tabular}

\section{Quantitative biofilm production}

The results of quantitative biofilm production test is shown in Table 2 as mean OD 570 values. According to OD values among $77 \%$ biofilm producers, 50 isolates (71\%) were categorized strong biofilm former (OD>0.204), 16 $(20 \%)$ as moderate biofilm former $(0.102<0 D<0.204)$ and 11 isolates $(14 \%)$ as weak biofilm former $(0.0551<\mathrm{OD}<0.102)$. There are a significant between MDR phenotype, biofilm formation and efflux pump among K. pneumoniae strains $(P<0.05)$. Moreover, there was a significant correlation between biofilm formation in isolates recovered from urine comparing to other type specimens $(P<0.05)$.

\section{Frequency of biofilm, efflux pump and virulence associated genes}

The prevalence of biofilm, efflux pump and virulence associated genes are given in Table 3. The $m r k A, m r k D$ and $\mathrm{fimH}$ genes encoding type 1 and type 3 fimbrial adhesion engage in biofilm formation were present in all biofilm former strains. Moreover, the efflux pump genes including $A c r A B, T o / C$ and $m d t K$ were observed 41 (41\%), $33(33 \%)$ and $26(26 \%)$ strains, respectively. The AcrAB was more prevalent in K. pneumoniae strains comparing to other efflux pump genes. In addition, the AcrAB efflux pump gene was more prevalent in urine samples comparing to other clinical specimens.

In addition, the virulence related genes including enterobactin biosynthesis gene (entB), outer membrane protein coding gene (traT) and mucoid phenotype A (rmpA) was seen in $80(80 \%), 62(62 \%)$ and $48(48 \%)$ strains, 
respectively. As reported in Table 3, analysis of selected genes showed that biofilm was more pronounced among virulence associated gene positive than among negative strains. The entB virulence gene was detected in all blood, CSF and sputum isolates. There were 8 virulence profiles (V1-V8) Based on virulence detected gene and $\mathrm{V} 1$ was the most prevalent virulence type. 
Table 3

Biofilm, efflux pump and virulence association genes among K. pneumoniae strains.

\begin{tabular}{|c|c|c|c|c|c|c|c|c|c|c|c|}
\hline \multirow[t]{2}{*}{$\begin{array}{l}\text { Isolate } \\
\text { NO }\end{array}$} & \multicolumn{3}{|c|}{$\begin{array}{l}\text { Biofilm association } \\
\text { genes }\end{array}$} & \multicolumn{3}{|c|}{ Efflux pump genes } & \multicolumn{3}{|c|}{$\begin{array}{l}\text { Virulence association } \\
\text { gene }\end{array}$} & \multirow{2}{*}{$\begin{array}{l}\text { Virulence } \\
\text { gene } \\
\text { Type }\end{array}$} & \multirow[t]{2}{*}{$\begin{array}{l}\text { Type of } \\
\text { samples }\end{array}$} \\
\hline & $m r k A$ & $m r k D$ & fimH & $A C r A B$ & TolC & $m d t K$ & entB & $\operatorname{TraT}$ & $r m p A$ & & \\
\hline 1 & + & + & + & - & + & - & + & + & - & V1 & Urine \\
\hline 2 & + & + & + & + & + & + & + & + & + & V2 & Urine \\
\hline 3 & + & + & + & - & + & - & + & + & + & V2 & Urine \\
\hline 4 & + & + & + & + & - & - & + & + & + & V2 & Urine \\
\hline 5 & + & + & + & + & - & + & - & + & - & V3 & Urine \\
\hline 6 & + & + & + & - & - & + & + & + & - & V1 & Urine \\
\hline 7 & + & + & + & - & + & - & + & - & + & V4 & Urine \\
\hline 8 & + & + & + & + & - & + & + & - & + & V4 & Urine \\
\hline 9 & + & + & + & - & - & - & - & + & + & V5 & Urine \\
\hline 10 & + & + & + & - & + & + & + & + & - & V1 & Urine \\
\hline 11 & + & + & + & + & + & - & + & + & - & V1 & Urine \\
\hline 12 & + & + & + & - & - & - & + & + & - & V1 & Urine \\
\hline 13 & + & + & + & - & + & - & + & + & - & V1 & Urine \\
\hline 14 & + & + & + & - & - & - & - & + & - & V3 & Urine \\
\hline 15 & + & - & - & + & + & - & + & - & + & V4 & Urine \\
\hline 16 & - & + & + & - & - & + & + & + & + & V2 & Urine \\
\hline 17 & + & + & + & - & - & + & + & + & + & V2 & Urine \\
\hline 18 & + & + & + & + & + & + & + & + & + & V2 & Urine \\
\hline 19 & - & + & + & - & - & - & - & + & - & V3 & Urine \\
\hline 20 & + & - & - & + & - & - & + & + & - & V1 & Urine \\
\hline 21 & + & + & + & - & - & - & + & + & - & V1 & Urine \\
\hline 22 & + & + & + & + & + & - & - & + & - & V3 & Urine \\
\hline 23 & + & + & + & - & - & - & + & + & - & V1 & Urine \\
\hline 24 & + & + & + & + & - & - & - & - & + & V6 & Urine \\
\hline 25 & + & + & + & - & + & - & + & + & + & V2 & Urine \\
\hline 26 & + & + & + & - & + & - & - & + & + & V5 & Urine \\
\hline 27 & + & + & + & - & - & - & - & + & - & V3 & Urine \\
\hline
\end{tabular}




\begin{tabular}{|c|c|c|c|c|c|c|c|c|c|c|c|}
\hline 28 & - & + & + & - & + & - & + & + & - & V1 & Urine \\
\hline 29 & - & - & - & + & - & - & + & + & + & V2 & Urine \\
\hline 30 & + & - & - & - & - & - & - & + & + & V5 & Urine \\
\hline 31 & + & + & + & + & - & - & + & + & + & V2 & Urine \\
\hline 32 & + & - & - & + & - & + & - & - & - & V7 & Urine \\
\hline 33 & - & + & + & + & - & + & + & + & - & V1 & Urine \\
\hline 34 & + & + & + & - & + & - & + & + & - & V1 & Urine \\
\hline 35 & - & + & + & - & - & - & - & + & - & V3 & Urine \\
\hline 36 & - & + & + & + & - & - & + & + & - & V1 & Urine \\
\hline 37 & + & - & - & + & - & - & - & + & - & V3 & Urine \\
\hline 38 & + & - & - & + & - & - & + & + & - & V1 & Urine \\
\hline 39 & + & + & + & + & + & - & + & + & - & V1 & Urine \\
\hline 40 & + & + & + & - & - & + & + & - & - & V8 & Urine \\
\hline 41 & + & + & + & - & - & - & + & - & + & V4 & Urine \\
\hline 42 & + & + & + & - & - & - & + & - & + & V4 & Urine \\
\hline 43 & + & + & + & - & - & + & + & - & + & V4 & Urine \\
\hline 44 & + & + & + & + & + & - & - & - & + & V6 & Urine \\
\hline 45 & + & + & + & + & + & - & + & + & + & V2 & Urine \\
\hline 46 & + & + & + & + & + & - & + & + & + & V2 & Urine \\
\hline 47 & + & + & + & - & - & + & + & + & - & V1 & Urine \\
\hline 48 & + & + & + & - & - & - & + & - & - & V8 & Urine \\
\hline 49 & + & + & + & - & + & + & - & - & - & V7 & Urine \\
\hline 50 & + & - & - & + & - & + & + & - & + & V4 & Urine \\
\hline 51 & - & + & + & - & + & - & + & + & + & V2 & Blood \\
\hline 52 & + & + & + & + & + & - & + & + & + & V2 & Urine \\
\hline 53 & + & + & + & + & - & + & + & + & + & V2 & Sputum \\
\hline 54 & + & + & + & - & + & - & + & - & + & V6 & Blood \\
\hline 55 & + & + & + & - & - & - & + & - & + & V4 & Blood \\
\hline 56 & + & + & + & + & - & + & + & - & + & V4 & Blood \\
\hline 57 & + & + & + & - & - & - & - & - & + & V4 & Urine \\
\hline 58 & + & + & + & - & + & + & + & + & - & V1 & Blood \\
\hline
\end{tabular}




\begin{tabular}{|c|c|c|c|c|c|c|c|c|c|c|c|}
\hline 59 & + & + & + & - & - & - & - & + & - & V3 & Urine \\
\hline 60 & + & + & + & - & + & + & + & + & - & V1 & Urine \\
\hline 61 & + & + & + & - & - & - & + & + & + & V2 & Blood \\
\hline 62 & + & + & + & - & - & - & + & + & + & V2 & CSF \\
\hline 63 & + & + & + & - & + & + & - & - & + & V6 & Urine \\
\hline 64 & + & + & + & - & + & + & + & - & + & V4 & Sputum \\
\hline 65 & + & + & + & - & - & - & + & - & + & V4 & Blood \\
\hline 66 & + & + & + & - & - & - & + & - & - & V8 & Sputum \\
\hline 67 & + & + & + & - & + & - & - & - & - & v7 & Urine \\
\hline 68 & + & + & + & - & + & - & + & + & - & V1 & Blood \\
\hline 69 & + & + & + & + & - & - & + & + & - & V1 & Urine \\
\hline 70 & + & + & + & + & - & - & + & + & + & $\mathrm{V} 2$ & Blood \\
\hline 71 & + & + & + & + & - & - & - & - & - & V7 & Urine \\
\hline 72 & + & + & + & - & - & - & + & - & - & V8 & CSF \\
\hline 73 & + & + & + & + & - & - & + & - & + & V4 & Urine \\
\hline 74 & + & + & + & - & - & - & - & + & - & $\mathrm{V} 1$ & Urine \\
\hline 75 & + & + & + & + & + & - & + & + & - & V3 & Blood \\
\hline 76 & + & + & + & + & + & - & + & - & + & V4 & Urine \\
\hline 77 & - & + & + & - & - & - & + & + & - & $\mathrm{V} 1$ & Blood \\
\hline 78 & + & + & + & - & - & - & + & - & - & V8 & Urine \\
\hline 79 & + & + & + & - & - & - & + & - & - & V8 & Blood \\
\hline 80 & + & + & + & + & - & - & + & - & - & V8 & Blood \\
\hline 81 & + & + & + & - & - & - & + & + & + & V2 & CSF \\
\hline 82 & + & + & + & + & - & - & + & - & - & V8 & Urine \\
\hline 83 & + & + & + & - & - & - & + & - & - & V8 & Blood \\
\hline 84 & + & + & + & - & - & - & + & + & - & $\mathrm{V} 1$ & Urine \\
\hline 85 & + & + & + & + & - & - & + & - & - & V8 & Blood \\
\hline 86 & - & + & + & + & - & - & + & - & - & V8 & Sputum \\
\hline 87 & + & + & + & - & - & + & + & + & - & $\mathrm{V} 1$ & Blood \\
\hline 88 & - & + & + & - & - & + & + & - & - & V8 & Blood \\
\hline 89 & + & - & - & + & - & - & + & - & + & V4 & Urine \\
\hline
\end{tabular}




\begin{tabular}{|llllllllllll|}
90 & + & - & - & + & - & - & + & + & + & V2 & Sputum \\
91 & + & - & - & + & - & - & + & + & + & V2 & Urine \\
92 & + & + & + & - & - & - & + & - & - & V8 & Urine \\
93 & + & + & + & - & - & + & + & - & - & V8 & Blood \\
94 & - & + & + & - & - & - & + & - & + & V4 & Urine \\
95 & + & - & - & + & - & - & + & + & - & V1 & Urine \\
96 & + & + & + & - & + & + & + & + & + & V2 & Blood \\
97 & + & + & + & + & - & - & + & + & - & V1 & Urine \\
98 & + & + & + & - & - & - & + & + & + & V2 & CSF \\
99 & + & + & + & + & - & - & + & + & + & V2 & Blood \\
100 & + & + & + & - & - & + & + & - & - & V8 & Sputum \\
\hline
\end{tabular}

\section{AcrAB efflux pump and mrkA biofilm gene expression}

The eight MDR $(1,6,10,13,17,18,23$ and 25 isolates NO.) and non-MDR (30, 37, 38, 46, 89, 90, 91 and 95 isolates NO.) selected $K$. pneumoniae strains were used for $A c r A$ and $m r k A$ gene expression analysis. The results of Real Time PCR showed that $A c r A$ and $m r k A$ gene were up-regulated significantly in MDR isolates comparing to non-MDR isolates. There was a significant relationship between MDR isolates, AcrA and $m r k A$ gene expression $(\mathrm{P}<0.05)$ (Figure 2).

\section{Rep-PCR typing}

According to the dendrogram, Repetitive element sequence-based PCR (rep-PCR) revealed 11 distinct patterns of K. pneumoniae isolates (Figure 3). The 11 rep genotypes were designed rep1 to rep11. The rep type 4-7 were the most common and, followed by type rep 1, 9 and 3, which consisted non-MDR isolates (Group I: 30, 95, 90, 91, Group C: 37,89 , Group J: 38 and 46 isolates NO). The rep 5 type was unique and each contained one strain exclusively. Based on statistical correlation tests, the rep 2, 4, 5, 6 and 7 significantly correlates with MDR strains and virulence patterns $(P<0.05)$. The strains showed high similarity which may suggest that those isolates consist a clonal lineage $(P<0.05)$.

\section{Discussion}

Multidrug-resistant (MDR) K. pneumoniae strains are an important cause of several life-treating infections, worldwide [32]. The extensive use of antimicrobial agents led to a high prevalence of MDR K. pneumoniae strains [33]. The increasing rate of $K$. pneumoniae strains resistant to multiple antimicrobials is a global public 
health problem [34]. In this study, the prevalence of MDR K. pneumoniae isolates was $92 \%$. The high rate of MDR strains was also shown in other studies. Moreover, $48 \%$ and $47 \%$ of $\mathrm{K}$. pneumoniae strains were resistant to imipenem and meropenem, respectively. From the results, it can be concluded that there has been a significant increase in carbapenem resistant $K$. pneumoniae isolates in Iran. It seems that the production of carbapenemase and metallobetalactamase have an important role in carbepenems resistance [35]. Our results is supported by some previous reports. Manjula et al, indicted $90.2 \%$ of isolates were MDR and the majority of MDR strains were resistant to a high range of antibiotics including penicillin, cephalosporin, fluoroquinolone, aminoglycoside, and sulfonamide [36].

One of the possible reason for high rate of antimicrobial resistance is lack of strict policies for use of antibiotics in Iran. Another mechanism of multidrug resistant is efflux pumps which used by $K$. pneumoniae strains [37]. The efflux pumps could reduce the intracellular concentration of antibiotics which is as an important cause of bacterial survival [38]. In our study, the AcrAB efflux pump is most common efflux pump in $K$. pneumoniae strains comparing to $m d t k$ and it was significantly correlated with MDR phenotype. Our results are consistent with other reports which indicated that the multidrug efflux pump system (AcrAB-TolC) in K. pneumoniae strains is responsible for antibiotics especially fluoroquinolones such as ciprofloxacin, tetracycline and beta-lactam antibiotics in MDR isolates.

In addition, $77 \%$ of $K$. pneumoniae strains were biofilm former and $89 \%$ of biofilm former were MDR. Until now, it has been shown a significant correlation between MDR phenotype and the biofilm forming ability of $K$. pneumoniae strains [39]. In addition, the relationship between antibiotic resistance and biofilm formation has also been studied in strains grown under the antibiotic dose especially in sub-minimum inhibitory concentration [40]. There was a significant relationship between strains of $K$. pneumoniae isolated from urine and biofilm formation. Most of urine originate strains exhibited strong biofilm capacity. In the current study, K. pneumoniae strains isolated from clinical specimens harbored high prevalence of efflux pump, biofilm and virulence associated genes. The virulence associated genes were also dominant in MDR strains. Type 1 fimbriae (fimH-1) and Type 3 fimbrial adhesion ( $m r k A$ and $m r k D$ ) are the most common bacteria cells adhesive agent which can mediate the binding of K. pneumoniae strains to endothelial and epithelial cells of urinary tracts cause to urinary tract infections $[41,42]$. Type 3 fimbrial adhesion plays an important role in the biofilm formation of $\mathrm{K}$. pneumoniae strains, however, their exact role in the biofilm formation remains unclear [43]. The results of Nirwati et al, 2019 study on biofilm formation in K. pneumoniae strains isolated from clinical samples showed that $85.63 \%$ of the strains were biofilm and that is more than the results of our study [44]. In our study, the fimH, mrkA and mrkD were detected in all types of urine, blood and CSF isolates especially in urine isolates. The enterobactin biosynthesis gene (entB), serum resistance-associated outer membrane lipoprotein (traT) and regulators of mucoid phenotype $\mathrm{A}(\mathrm{rmpA})$ were detected in $80 \%, 62 \%$ and $48 \%$ of $\mathrm{K}$. pneumonia strains. The traT gene encodes an outer membrane protein which plays an important role in conjugation and inhibition of complement cascade and act as invasin [45]. Molecular typing of bacteria is a very suitable method for differentiation of microbial pathogens and among the typing methods, rep-PCR technique is widely used for genotyping of bacterial strains. [46]. In our study, out of $100 \mathrm{~K}$. pneumoniae strains, rep-PCR could differentiated into 11 distinct patterns and most MDR strains were put in the same patterns. Our data confirmed the Lai et al results which showed pathogenic $K$. pneumoniae strains are heterogeneous, because of variation in genome sequences [47]. Our results showed the correlation of MDR strains with rep-PCR patterns. However, the rep-PCR revealed no statistically significant correlation with virulence type. In addition, the rep-PCR results showed that 
the same rep-type in two studied hospitals indicated that the same clonal distribution of $K$. pneumoniae in two hospitals. Our findings can help to interpretation of MDR K. pneumoniae outbreaks associated with specific patterns in the future.

\section{Conclusion}

In our study, we reported the high prevalence of MDR K. pneumoniae strains with resistance to multiple antimicrobial agents, the ability to formed biofilm and presence of efflux pump, biofilm and virulence associated genes which can be a great barrier to treatment of $K$. pneumoniae related infections. Moreover, high genetic similarity of MDR strains in hospitals showed clonal dissemination of $K$. pneumoniae strains that requiring control tools. However, further studies are needed to investigate other epidemiological aspects of the K. pneumoniae strains.

\section{Abbreviations}

RND: Resistance Nodulation Division

MDR: multidrug-resistant

rep-PCR: Repetitive element sequence-based PCR

CSF: cerebrospinal fluid

MR-VP: (methyl red - Voges Proskauer)

CLSI: Clinical and Laboratory Standards Institute

\section{Declarations}

\section{Availability of data and materials}

All quantitative data generated or analysed during this study are included in this published article.

\section{Author information}

Molecular Biology Research Center, System Biology and Poisonings Institute, Baqiyatallah University of Medical Sciences, Tehran, Iran

Amir Mirzaie, Reza Ranjbar

\section{Contributions}

R. R conceive and designed the experiments. A. A performed the experiments, analyzed the results, done the statistical analyses and wrote the manuscript. All authors read and approved the final manuscript. 


\section{Corresponding author}

Correspondence to Reza Ranjbar

\section{Ethics declarations}

\section{Ethics approval and consent to participate}

Ethics approval for this study was obtained from Baqiyatallah University of Medical Sciences, Tehran, Iran permitted this study with number: IR.BMSU.REC.1397.056. Written informed consent was obtained from all patients participating in this study.

\section{Consent for publication}

Not applicable.

\section{Conflict of Interest}

The authors declare that there are no conflict of interest.

\section{Funding}

This study was supported by the ministry of health and medical education, Tehran, Iran.

\section{Acknowledgments}

Research reported in this publication was supported by a grant provided from the ministry of health and medical education, Tehran, Iran.

\section{References}

1. José A Bengoechea, Joana Sa Pessoa. Klebsiella pneumoniae infection biology: living to counteract host defences. FEMS Microbiol Rev. 2019; 43(2): 123-144.

2. Ranjbar R, Fatahian Kelishadrokhi A, Chehelgerdi M. Molecular characterization, serotypes and phenotypic and genotypic evaluation of antibiotic resistance of the Klebsiella pneumoniae strains isolated from different types of hospital-acquired infections. Infect Drug Resist. 2019; 12: 603-611.

3. Kelly L. Wyres, Ryan R. Wick, Louise M. Judd, Roni Froumine, Alex Tokolyi, Claire L. Gorrie, Margaret M. C. Lam, Sebastián Duchêne, Adam Jenney, Kathryn E. Holt. Distinct evolutionary dynamics of horizontal gene transfer in drug resistant and virulent clones of Klebsiella pneumoniae. PLoS Genet. 2019; 15(4): e1008114. 
4. Ciccozzi M, Cella E, Lai A, De Florio L, Antonelli F, Fogolari M, Maria Di Matteo F, Pizzicannella M, Colombo B, Dicuonzo G, Angeletti S. Phylogenetic analysis of multi-drug resistant Klebsiella pneumoniae strains from Duodenoscope biofilm: microbiological surveillance and reprocessing improvements for infection prevention. Front Public Health. 2019; 7: 219. Published online 2019. doi: 10.3389/fpubh.2019.00219

5. Fuzi M, Baño JR, Toth A. Global Evolution of Pathogenic Bacteria With Extensive Use of Fluoroquinolone Agents. Front Microbiol. 2020; 11: 271. Published online 2020. doi: 10.3389/fmicb.2020.00271

6. Wanjiang Z, Yao Z, Changzhen W, Wenyu L, Ruichao L, Fuguang C, Tian L, Yanhe Zh, Stefan S, Siguo L. Characterization of a multidrug-resistant porcine Klebsiella pneumoniae sequence type 11 strain coharboring b/a $a_{\mathrm{KPC}-2}$ and fosA3 on two novel hybrid plasmids. mSphere. 2019; 4(5): e00590-19.

7. Yoon EJ, Oh Y, Jeong SH. Development of tigecycline resistance in carbapenemase-producing Klebsiella pneumoniae sequence type 147 via AcrAB overproduction mediated by replacement of the ramA promoter. Ann Lab Med. 2020; 40(1):15-20.

8. Xu Q, Jiang J, Zhu Z, Xu T, Sheng ZK, Ye M, Xu X, Wang M. Efflux pumps AcrAB and OqxAB contribute to nitrofurantoin resistance in an uropathogenic Klebsiella pneumoniae Int J Antimicrob Agents. 2019;54(2):223-227.

9. Naha S, Sands K, Mukherjee S, Roy C, Rameez MJ, Saha B, Dutta S, Walsh TR, Basu S. KPC-2-producing Klebsiella pneumoniae ST147 in a neonatal unit: Clonal isolates with differences in colistin susceptibility attributed to AcrAB-TolC pump. Int J Antimicrob Agents. 2020; 55(3):105903.

10. Yoon EJ, Oh Y, Jeong SH. Development of tigecycline resistance in carbapenemase-producing Klebsiella pneumoniae sequence type 147 via AcrAB overproduction mediated by replacement of the ramA promoter. Ann Lab Med. 2020;40(1):15-20.

11. Grimsey EM, Fais C, Marshall RL, Ricci V, Ciusa ML, Stone JW, Ivens A, Malloci G, Ruggerone P, Vargiu AV, Piddock LJV. Chlorpromazine and amitriptyline are substrates and inhibitors of the AcrB multidrug efflux pump. 2020;11(3):e00465-20.

12. Shao L, Yao B, Yang J, Li X, Ye K, Zhang Y, Wang C. Characterization of a multidrug-resistant Klebsiella pneumoniae ST3330 clone responsible for a nosocomial outbreak in a neonatal intensive care unit. Ann Palliat Med. 2020; 9(3):1092-1102.

13. Sahoo RK, Das A, Gaur M, Pattanayak A, Sahoo S, Debata NK, Rahman PKSM, Subudhi E. Genotypic validation of extended-spectrum $\beta$-lactamase and virulence factors in multidrug resistance Klebsiella pneumoniae in an Indian hospital. Pathog Glob Health. 2019; 113(7):315-321.

14. Marques C, Belas A, Aboim C, Cavaco-Silva P, Trigueiro G, Gama LT, Pomba C. Evidence of sharing of Klebsiella pneumoniae strains between healthy companion animals and cohabiting humans. J Clin Microbiol. 2019; 57(6):e01537-18.

15. Tang $M$, Wei $X$, Wan X, Ding Z, Ding Y, Liu The role and relationship with efflux pump of biofilm formation in Klebsiella Pneumoniae. Microb Pathog. 2020;147:104244.

16. Vuotto C, Longo F, Pascolini C, Donelli G, Balice MP, Libori MF, Tiracchia V, Salvia A, Varaldo PE. Biofilm formation and antibiotic resistance in Klebsiella pneumoniae urinary strains. J Appl Microbiol. 2017; 123(4):1003-1018.

17. Subramanian P, Shanmugam N, Sivaraman U, Kumar S, Selvaraj S. Antiobiotic resistance pattern of biofilm-forming uropathogens isolated from catheterized patients in Pondicherry, India. Australas Med J. 
2012; 5: 344-348.

18. Highsmith AK, Jarvis WR. Klebsiella pneumoniae. selected virulence factors that contribute to pathogenicity. Infect Control. 1985; 6(2):75-7.

19. Ghalavand Z, Heidary Rouchi A, Bahraminasab H, Ravanasa E, Mirsamadi ES, Nodeh Farahani N, Nikmanesh B. Molecular testing of Klebsiella pneumoniae contaminating tissue allografts recovered from deceased donors. Cell Tissue Bank. 2018;19(3):391-398.

20. Mukherjee S, Bhattacharjee A, Naha S, Majumdar T, Debbarma SK, Kaur H, Dutta S, Basu S. Molecular characterization of NDM-1-producing Klebsiella pneumoniae ST29, ST347, ST1224, and ST2558 causing sepsis in neonates in a tertiary care hospital of North-East India. Infect Genet Evol. 2019; 69:166-175.

21. Collee J. In Mackie \& Mccartney Practical Medical Microbiology(ed Collee J. G., Fraser A. G., Marimon B. P., Simmons A.) (Elsevier, 2007).

22. (Clinical and Laboratory Standards Institute, PA, USA, 2020).

23. Behdad R, Pargol M, Mirzaie A, Karizi SZ, Noorbazargan H, Akbarzadeh I. Efflux pump inhibitory activity of biologically synthesized silver nanoparticles against multidrug-resistant Acinetobacter baumannii clinical isolates. J Basic Microbiol. 2020; 60(6):494-507.

24. Hasan ME, Shahriar A, Shams F, Nath AK, Emran TB. Correlation between biofilm formation and antimicrobial susceptibility pattern toward extended spectrum $\beta$-lactamase (ESBL)- and non-ESBLproducing uropathogenic bacteria. J Basic Clin Physiol Pharmacol. 2020 Jan 13:/j/jbcpp.ahead-ofprint/jbcpp-2019-0296/jbcpp-2019-0296.xml. doi: 10.1515/jbcpp-2019-0296.

25. Alkhudhairy MK, Alshadeedi SMJ, Mahmood SS, Al-Bustan SA, Ghasemian A. Comparison of adhesin genes expression among Klebsiella oxytoca ESBL-non-producers in planktonic and biofilm mode of growth, and imipenem sublethal exposure. Microb Pathog. 2019; 134:103558.

26. Wasfi R, Elkhatib WF, Ashour HM. Molecular typing and virulence analysis of multidrug resistant Klebsiella pneumoniae clinical isolates recovered from Egyptian hospitals. Sci Rep. 2016; 6:38929.

27. SahlyH, Navon-Venezia S, L Roesler, A Hay, Y Carmeli, R Podschun, C Hennequin, C Forestier, I Ofek. Extended-spectrum beta-lactamase production is associated with an increase in cell invasion and expression of fimbrial adhesins in Klebsiella Pneumoniae. Antimicrob Agents Chemother. 2008; 52(9):302934.

28. El Fertas-Aissani R., Messai Y., Alouache S. \& Bakour R. Virulence profiles and antibiotic susceptibility patterns of Klebsiella pneumoniaestrains isolated from different clinical specimens. Pathol Biol (Paris) 61, 209-216, doi: 10.1016/j.patbio.2012.10.004 (2013).

29. Siu L. K. et al. Molecular typing and virulence analysis of serotype K1 Klebsiella pneumoniaestrains isolated from liver abscess patients and stool samples from noninfectious subjects in Hong Kong, Singapore, and Taiwan. Journal of Clinical Microbiology 49, 3761-3765, doi: 10.1128/JCM.00977-11 (2011).

30. Marroquin S, Brittney Gimza, Brooke Tomlinson, Michelle Stein, Andrew Frey, Rebecca A Keogh, Rachel Zapf, Daniel A Todd, Nadja B Cech, Ronan K Carroll, Lindsey N Shaw. MroQ is a novel Abi-Domain protein that influences virulence gene expression in Staphylococcus aureus via modulation of Agr activity. Infect Immun. 2019; 87(5):e00002-19. 
31. Mohamed M. Hassan \& El-Sayed B. Belal. Antibiotic resistance and virulence genes in enterococcus strains isolated from different hospitals in Saudi Arabia. 2016; 30:4, 726-732.

32. Petrosillo N, Taglietti F, Granata G. Treatment options for Colistin resistant Klebsiella pneumoniae: Present and Future. J Clin Med. 2019; 8(7):934.

33. Ciccozzi M, Cella E, Lai A, De Florio L, Antonelli F, Fogolari M, Di Matteo FM, Pizzicannella M, Colombo B, Dicuonzo G, Angeletti S. Phylogenetic analysis of multi-drug resistant Klebsiella pneumoniae strains From duodenoscope biofilm: microbiological surveillance and reprocessing improvements for infection prevention. Front Public Health. 2019; 7:219.

34. Khandelwal V, Sharma S. Fatal MDR Klebsiella in ICU - How was it Dealt with? Indian J Crit Care Med. 2019; 23(9):411-413.

35. Durante-Mangoni E, Andini R, Zampino R. Management of carbapenem-resistant Enterobacteriaceae infections. Clin Microbiol Infect. 2019; 25(8):943-950.

36. GM N, CM G. Nagshetty K, Patil SA, Gaddad SM, Shivannavar CT: antibiotic susceptibility pattern of ESbetaL producing Klebsiella pneumoniae isolated from urine samples of pregnant women in Karnataka. J Clin Diagn Res. 2014;8(10):DC08-DC11.

37. Maurya N, Jangra M, Tambat R, Nandanwar H. Alliance of efflux pumps with $\beta$-lactamases in multidrugresistant Klebsiella pneumoniae Microb Drug Resist. 2019; 25(8):1155-1163.

38. Xu Q, Jiang J, Zhu Z, Xu T, Sheng ZK, Ye M, Xu X, Wang M. Efflux pumps AcrAB and OqxAB contribute to nitrofurantoin resistance in an uropathogenic Klebsiella pneumoniae Int J Antimicrob Agents. 2019; 54(2):223-227.

39. Ostria-Hernandez ML, Juárez-de la Rosa KC, Arzate-Barbosa P, Lara-Hernández A, Sakai F, Ibarra JA, CastroEscarpulli G, Vidal JE. Nosocomial, Multidrug-Resistant Klebsiella pneumoniae Strains Isolated from Mexico City Produce Robust Biofilms on Abiotic Surfaces but Not on Human Lung Cells. Microb Drug Resist. 2018; 24(4):422-433.

40. Maharjan G, Khadka P, Siddhi Shilpakar G, Chapagain G, Dhungana GR. Catheter-Associated Urinary Tract Infection and Obstinate Biofilm Producers. Can J Infect Dis Med Microbiol. 2018; 2018:7624857.

41. Marques C, Menezes J, Belas A, Aboim C, Cavaco-Silva P, Trigueiro G, Telo Gama L, Pomba C. Klebsiella pneumoniae causing urinary tract infections in companion animals and humans: population structure, antimicrobial resistance and virulence genes. J Antimicrob Chemother. 2019; 74(3):594-602.

42. Panjaitan NSD, Horng YT, Cheng SW, Chung WT, Soo PC. EtcABC, a Putative Ell complex, regulates type 3 fimbriae via CRP-cAMP signaling in Klebsiella pneumoniae. Front Microbiol. 2019;10:1558.

43. Khalil MAF, Hager R, Abd-El Reheem F, Mahmoud EE, Samir T, Moawad SS, Hefzy EM. A Study of the Virulence Traits of Carbapenem-Resistant Klebsiella pneumoniae Isolates in a Galleria mellonella Model. Microb Drug Resist. 2019; 25(7):1063-1071.

44. Nirwati H, Kian Sinanjung, Fahrina Fahrunissa, Fernando Wijaya, Sarastia Napitupulu, Vania P Hati, Mohamad S Hakim, Andreanita Meliala, Abu T Aman, Titik Nuryastuti. Biofilm Formation and Antibiotic Resistance of Klebsiella pneumoniae Isolated From Clinical Samples in a Tertiary Care Hospital, Klaten, Indonesia. BMC Proc. 2019;13(Suppl 11):20. doi: 10.1186/s12919-019-0176-7.

45. Kuş H, Arslan U, Türk Dağı H, Fındık D. Investigation of various virulence factors of Klebsiella pneumoniae strains isolated from nosocomial infections. Mikrobiyol Bul. 2017; 51(4):329-339. 
46. Rojas LJ, Salim M, Cober E, Richter SS, Perez F, Salata RA, Kalayjian RC, Watkins RR, Marshall S, Rudin SD, Domitrovic TN, Hujer AM, Hujer KM, Doi Y, Kaye KS, Evans S, Fowler VG Jr, Bonomo RA, van Duin D. Colistin Resistance in Carbapenem-Resistant Klebsiella pneumoniae: Laboratory Detection and Impact on Mortality. Clin Infect Dis. 2017; 64(6):711-718.

47. Lai Y. C., Yang S. L., Peng H. L. \& Chang H. Y. Identification of genes present specifically in a virulent strain of Klebsiella pneumoniae. Infect Immun68, 7149-7151 (2000).

\section{Figures}

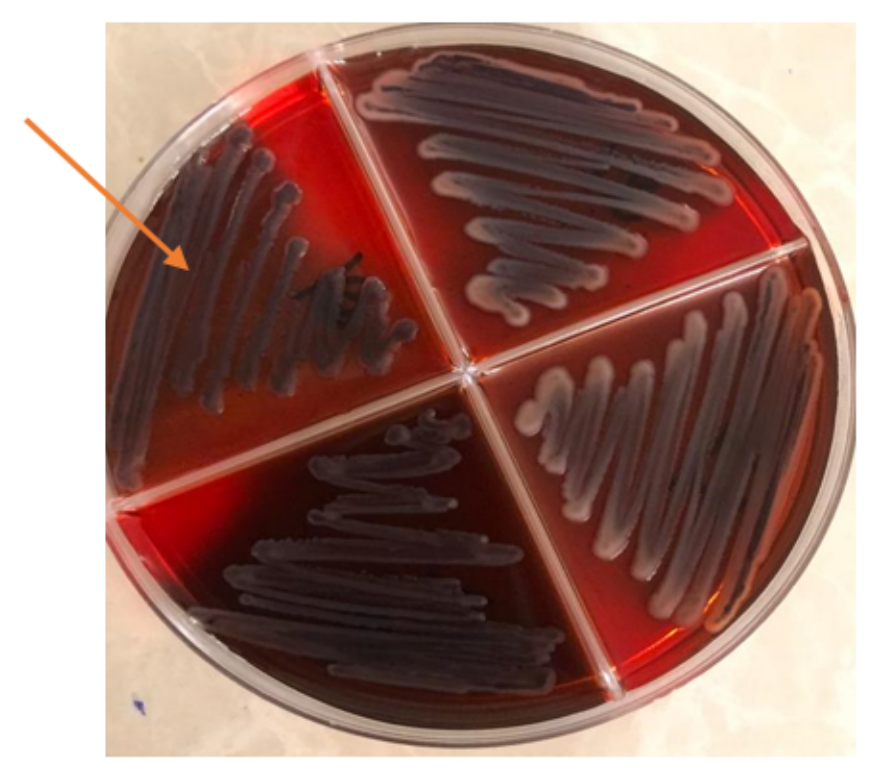

A

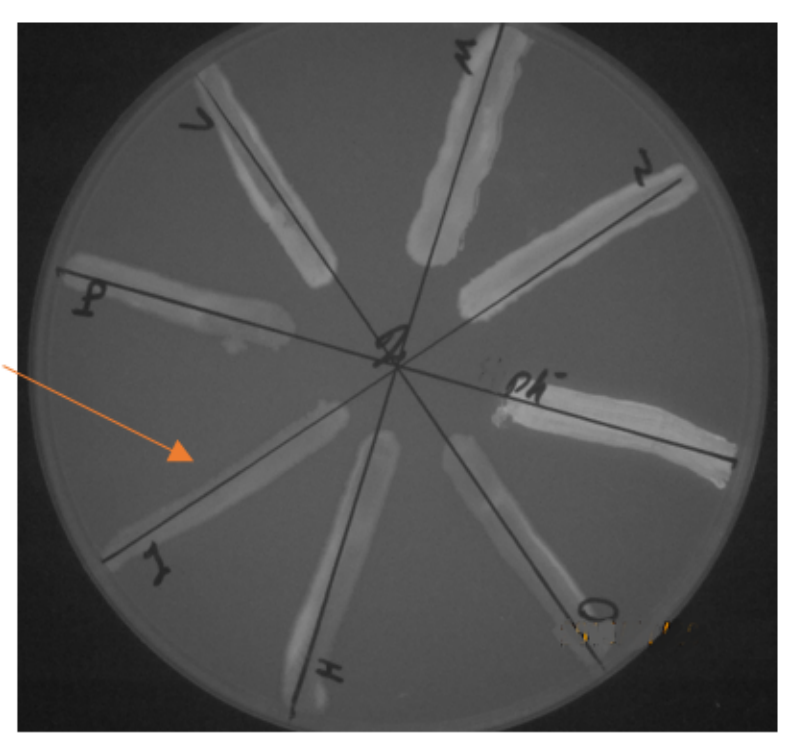

B

\section{Figure 1}

Congo red agar (A) and Cartwheel tests (B) for detection of biofilm producers and efflux pump system in $\mathrm{K}$. pneumoniae strains. As can be observed the biofilm forming bacteria exhibited the black appearance in colony (Arrow). Moreover, the isolates had efflux pump didn't show fluorescent emission under UV transilluminator (Arrow). 


\section{MDR isolates}

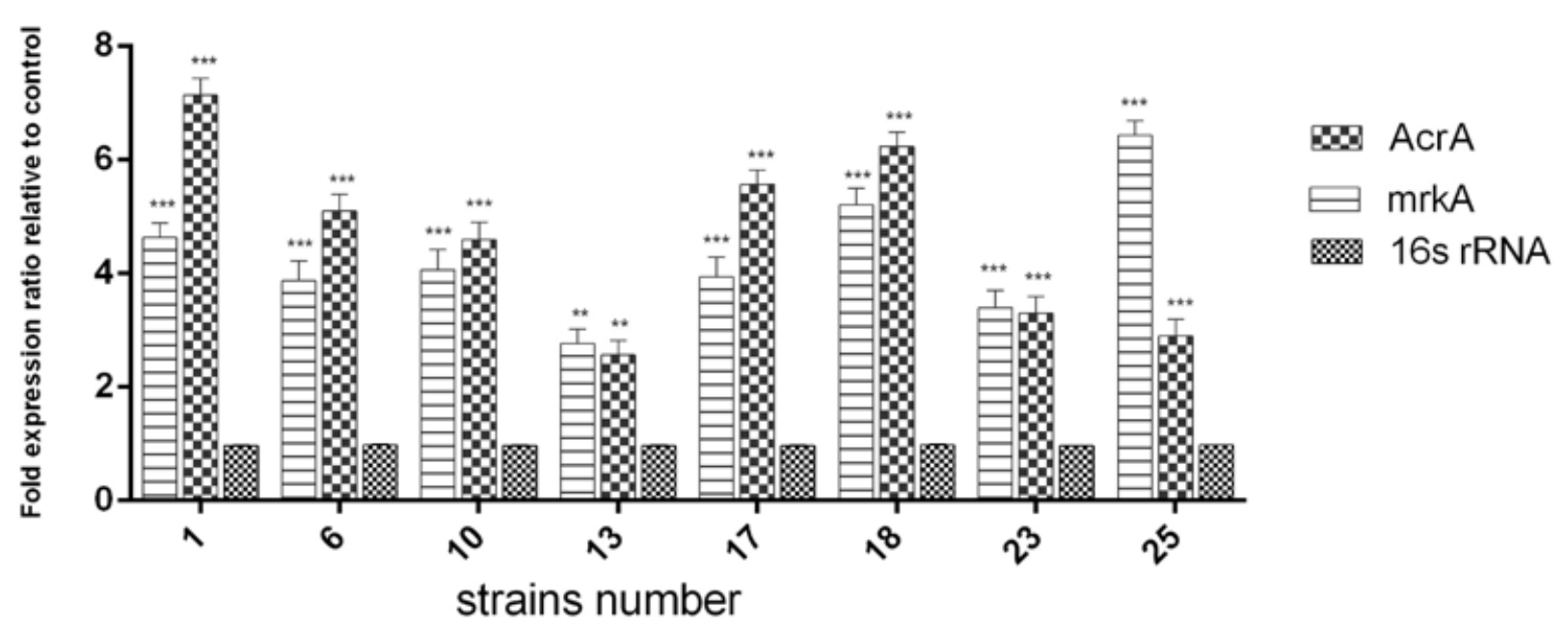

\section{Non-MDR isolates}

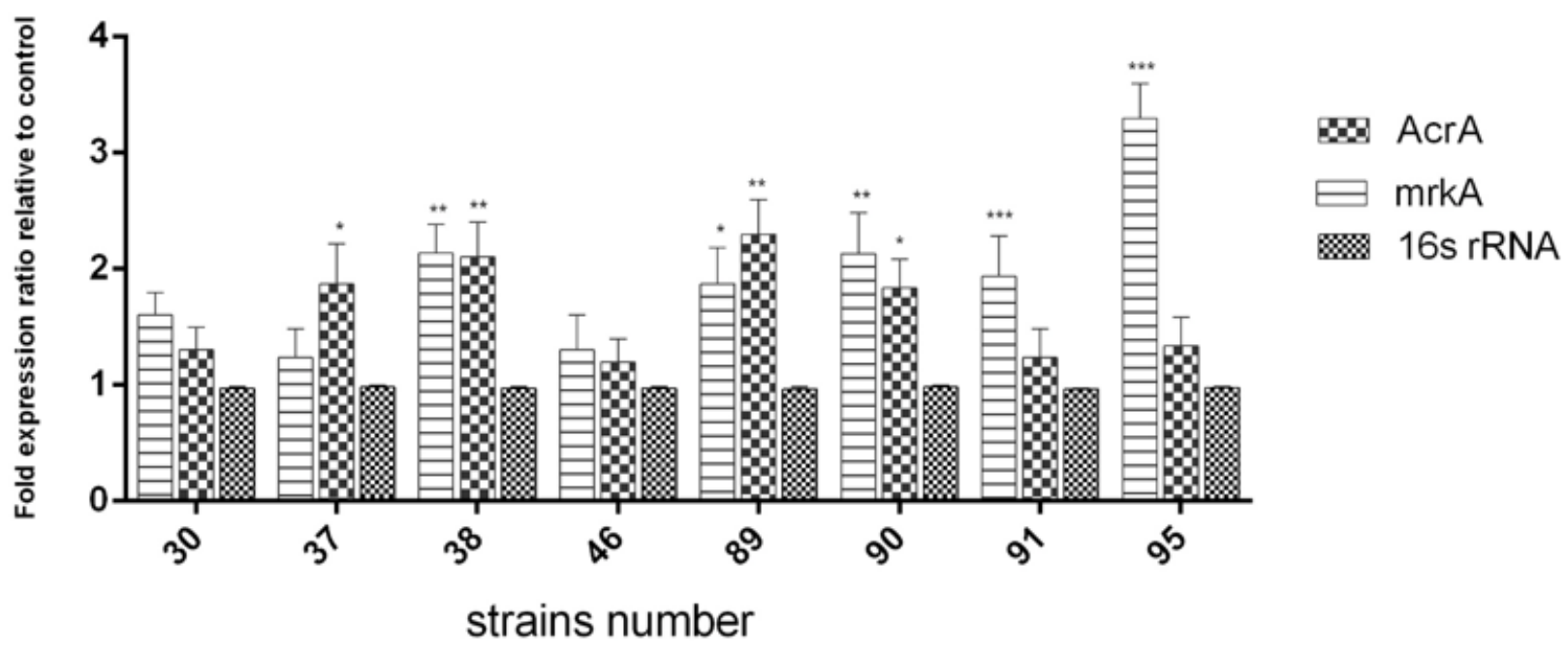

Figure 2

AcrA and mrkA relative gene expression among MDR and non-MDR strains as fold difference between AcrA, mrkA gene and 16S rRNA gene. Data are mean SD of three independent experiments. Significant difference specified as ${ }^{* \star *} \mathrm{p}<0.001,{ }^{\star *} \mathrm{p}<0.01,{ }^{*} \mathrm{p}<0.05$ 


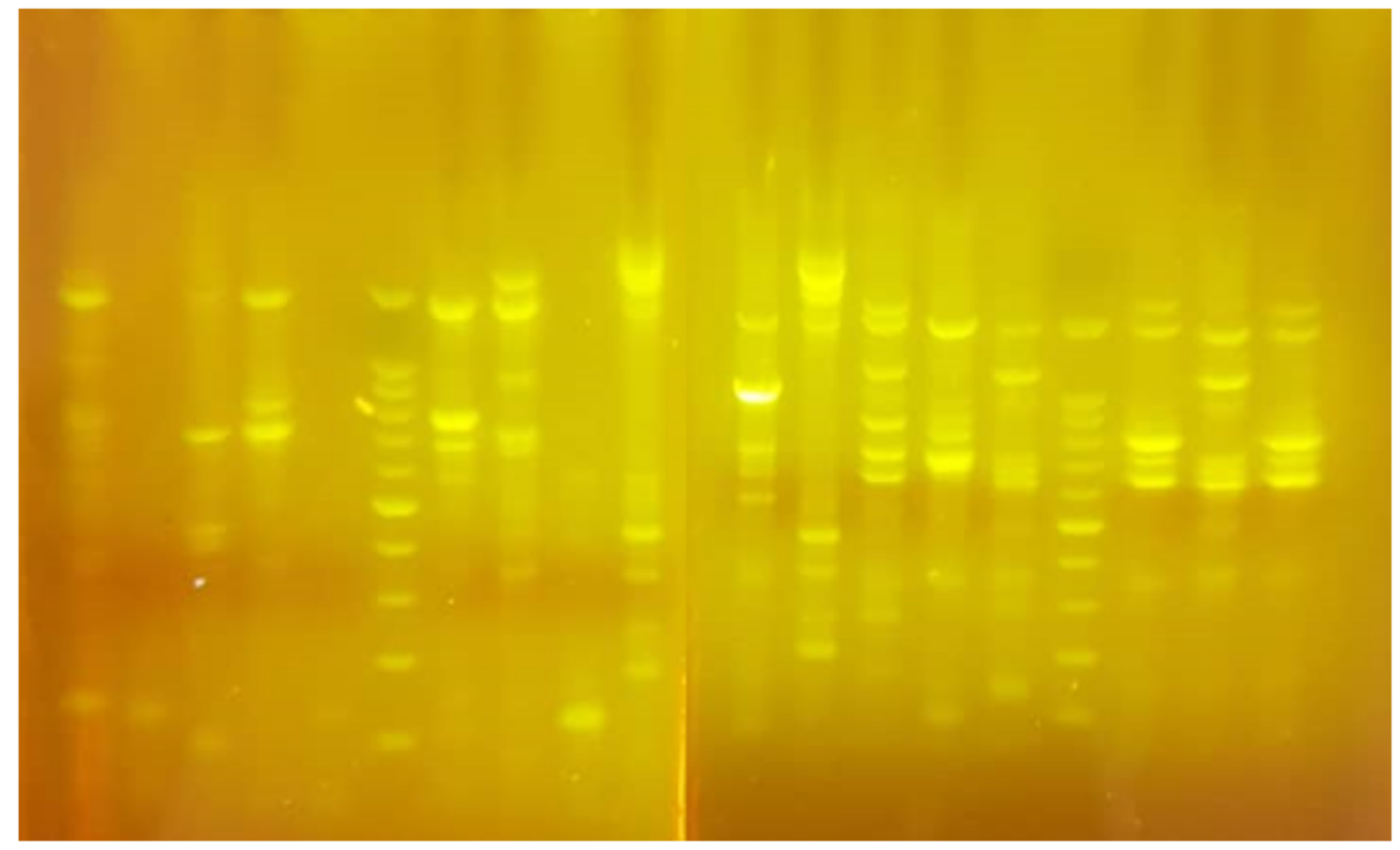

\section{Figure 3}

The rep-PCR fingerprints of K. pneumoniae strains.

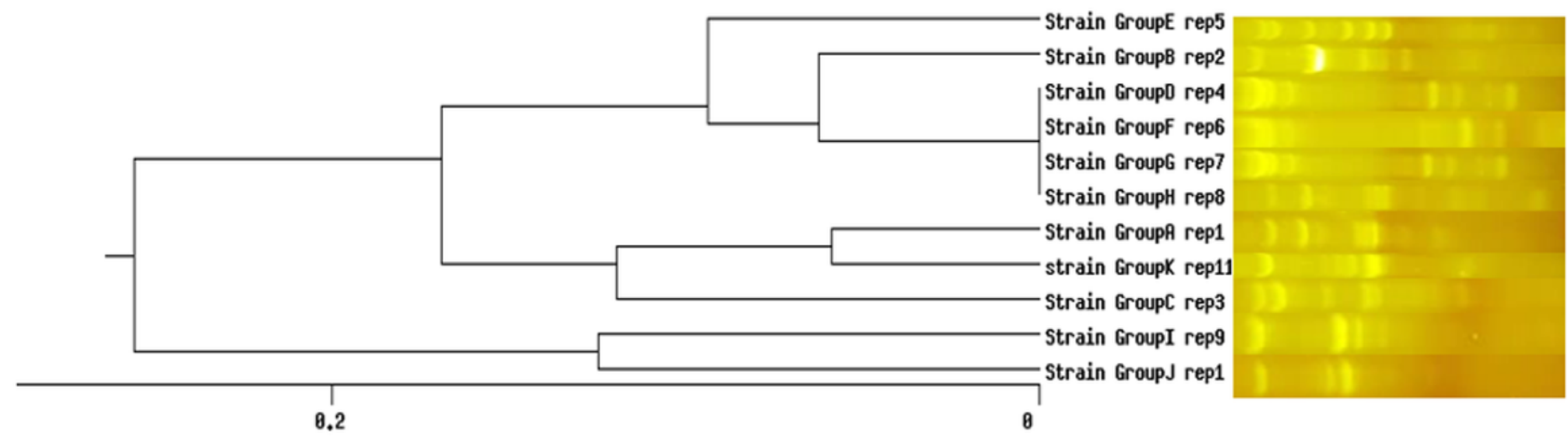

\section{Figure 4}

The 11 rep types identified by Rep-PCR in K. pneumoniae isolates using the UPGMA based on Dice similarity. 